\title{
Unexpected difficulties during intubation: there is always something new and it could happen to you
}

\begin{abstract}
A case report of an unexpected and unexplained difficult intubation due to limited mouth opening that did not exist preoperatively.
\end{abstract}

Volume 5 Issue 5 - 2016

\author{
Kotsimpogeorgou I, Athanasios Manoudis \\ Department of Anesthesiology, Larissa General Hospital, Greece \\ Correspondence: Athanasios Manoudis, Department of \\ Anesthesiology, Larissa General Hospital, Seferi 97-101 4I22I \\ Larissa, Greece, Tel +3069373II 053 \\ Email athanasios.man@gmail.com
}

Received: April 19, 2016 | Published: August 24, 2016

\section{Introduction}

Predicting difficult tracheal intubation is still a matter of debate among anesthesiologists, with each individual score or sign having a poor predictive value. ${ }^{1}$ In the present case report we present a case of difficult tracheal intubation that could not have been predicted with any score or clinical sign used for preoperative airway assessment.

\section{Case report}

A 39 year old woman ASA I, 75Kg was admitted for laparoscopic cholecystectomy. Preoperative assessment of airway revealed no abnormalities with a Mallampati I score with a mouth opening of at least $30 \mathrm{~mm}$. She referred no previous operations. After sedating the patient with intravenous midazolam $2 \mathrm{mg}$, anesthesia was induced using 200mg propofol and 50mg of rocuronium bromide and fentanyl $5 \mu \mathrm{g} / \mathrm{Kg}$. Mask ventilation was easy and it was performed for 90 seconds with $10 \mathrm{~L} / \mathrm{min}$ of oxygen and tracheal intubation was initiated. When manual pressure was applied to open the mouth, the opening decreased to about $15 \mathrm{~mm}$, making it impossible to insert a Machintosh 3 blade. Subsequently an Airtraq No 3 airway device was used but one attempt to intubate her was unsuccessful so an elastic gum bougie was used through the airtraq. A No 7 endotracheal tube was railroaded and the patient was successfully intubated. Immediately after intubation and 30 minutes later we measured again the mouth opening and it was still $15 \mathrm{~mm}$. Surgery was uneventful and anesthesia was maintained with desflurane $5 \%$ in $50 \% \mathrm{~N}_{2} \mathrm{O}$. After reversing the neuromuscular blockade using $4 \mathrm{mg} / \mathrm{kg}$ suggamadex, she was extubated and the mouth opening returned to the preoperative value.

\section{Discussion}

Searching Pubmed we found case reports of limited mouth opening only associated with masticatory muscle tendonaponeurosis hyperplasia (MMTAH), a disease characterized by limited mouth opening due to contracture of the masticatory muscles as a result of tendon hyperplasia and aponeuroses. ${ }^{2-4}$ in which the degree of mouth opening can markedly decrease after anesthesia induction. ${ }^{5}$ During the preoperative interview of the patient she did not refer anything and her clinical examination both preoperative and postoperative did not reveal any signs of MMTAH.

\section{Conclusion}

There is no other obvious explanation, and the message is clear: keep in mind that tracheal intubation may be unexpectedly difficult, for many reasons.

\section{Acknowledgments}

None.

\section{Conflicts of interest}

The authors declare there is no conflict of interests.

\section{Funding}

None.

\section{References}

1. Langeron O, Cuvillon P, Ibanez-Esteve C, et al. Prediction of difficult tracheal intubation. time for a paradigm change. Anesthesiology. 2012;117(6):1223-1233.

2. Yoda T, Sato T, Abe T, et al. Long-term results of surgical therapy for masticatory muscle tendon-aponeurosis hyperplasia accompanied by limited mouth opening. Int J Oral Maxillofac Surg. 2009;38(11):11431147.

3. Inoue S, Yamamoto I, Ikeda S, et al. Possible difficult laryngoscopy caused by masticatory muscle tendon-aponeurosis hyperplasia:we anesthesiologists should be aware of this disease. J Anesth. 2010;24(6):930-931.

4. Ikumi Y, Satoki I, Masahiko K, et al. Anesthetic Considerations for Masticatory Muscle Tendon-Aponeurosis Hyperplasia:A Report of 24 Cases. Anesth Prog. 2012;59(2):87-89.

5. Morimoto Y, Sugimura M, Hanamoto H, et al. Limited mouth opening following induction of anesthesia in two patients with masticatory muscle tendon-aponeurosis hyperplasia. J Clin Anesth. 2011;23(7):598600 . 